\title{
Age-Related Changes in Body Composition and Bone Mineral Density and Their Relationship with the Duration of Diabetes and Glycaemic Control in Type 2 Diabetes
}

This article was published in the following Dove Press journal:

Diabetes, Metabolic Syndrome and Obesity: Targets and Therapy

Ying Tang ',2
Lilin Gong'
Xiangjun Chen'
Zhipeng Du'
Jinbo Hu'
Zhixin Xu'
Jinshan Wu'
Qifu Li'
Zhihong Wang'
'Department of Endocrinology, The First
Affiliated Hospital of Chongqing Medical
University, Chongqing, People's Republic
of China; ${ }^{2}$ Department of Endocrinology,
West China-Guang'an Hospital, Sichuan
University, Sichuan, People's Republic of
China

Purpose: To investigate age-related changes in body composition $(\mathrm{BC})$ and bone mineral density (BMD) in type 2 diabetes (T2D) and analyse whether diabetes duration or glycaemic control affects these factors.

Patients and Methods: We enrolled 1474 hospitalized T2D patients (817 males and 657 females; 45-85 years). BC and BMD were assessed by dual-energy X-ray absorptiometry (DEXA). Patients were stratified into age groups: 45-54, 55-64, 65-74, and $\geq 75$ years. Continuous variables were compared using $t$-tests or one-way analysis of variance (ANOVA), and categorical variables were compared using chi-square tests. Effects of age, diabetes duration, and haemoglobin $\mathrm{A}_{1 \mathrm{C}}\left(\mathrm{HbA}_{1 \mathrm{c}}\right)$ on $\mathrm{BC}$ and $\mathrm{BMD}$ were assessed with multiple linear regression models.

Results: In T2D, in females, changes in fat mass index (FMI) were positively correlated with age, while changes in lean mass index (LMI) were unrelated to age. Changes in FMI or LMI in males were unrelated to age. For regional BC distribution, changes in visceral adipose tissue (VAT) were positively correlated with age for both males and females, while changes in appendage lean mass (ALM) were negatively correlated with age. For BMD, changes in total BMD (TBMD) in males were not correlated with age, while changes in lumbar spine BMD (LBMD) were positively correlated with age, and femoral neck BMD (FNBMD) was negatively correlated with age. Changes in BMD in all parts of females were negatively correlated with age. In addition, changes in $\mathrm{BC}$ and $\mathrm{BMD}$ were unrelated to diabetes duration, and $\mathrm{HbA}_{1 \mathrm{c}}$ was mainly associated with decreases in lean mass but had little effect on other $\mathrm{BC}$ and BMD parameters. Conclusion: In $\mathrm{T} 2 \mathrm{D}$, changes in $\mathrm{BC}$ and $\mathrm{BMD}$ were associated with age but not diabetes duration. A higher $\mathrm{HbA}_{1 \mathrm{c}}$ is associated with lower lean mass.

Keywords: type 2 diabetes, ageing, body composition, bone mineral density, dual-energy X-ray absorptiometry

\section{Introduction}

Ageing is associated with changes in body composition (BC) and bone mineral density (BMD), including the accumulation of fat mass (especially abdominal fat) and loss of lean mass and BMD. These changes are associated with increased disability and mortality. For example, fat regional distribution affects the relationship among obesity, metabolism and health and plays an important role in cardiovascular and metabolic diseases. ${ }^{1,2}$ Lean mass and BMD decreases result in an increased risk of oligomyopathy, osteoporosis and fracture in elderly individuals.
Correspondence: Zhihong Wang The First Affiliated Hospital of Chongqing Medical University, Yuzhong District, Chongqing, People's Republic of China $\mathrm{Tel} / \mathrm{Fax}+86-23-890 \mathrm{II} 552$

Email towzh7I3@I26.com 
In the general population, the Health, Aging, and Body Composition (Health ABC) Study and the Fels Longitudinal study are two of the largest studies to describe the pattern of age-related changes in $\mathrm{BC}$. The results showed that fat mass increases with age in both sexes, whereas lean mass declines. ${ }^{3-5}$ Human bone tissue begins to decline at approximately 40 years of age due to the dysfunction of osteoblasts and a relative increase in reabsorption by osteoclasts, which results in a decrease in BMD. A previous study in Chinese Han men over 50 years old reported that BMD in the femoral neck and total hip declined with age. ${ }^{6}$

Type 2 diabetes (T2D) is a highly prevalent metabolic disorder characterized by a state of insulin resistance and relative insulin deficiency. Many studies have shown that compared with the general population, T2D patients have more fat or an equivalent fat mass and lower lean mass. $^{7-10}$ Abdulameer et al ${ }^{11}$ summarised the studies on BMD in T2D patients and found that compared with the general population, most studies showed an increase in BMD in T2D patients, while some studies showed that BMD decreased or did not significantly change. T2D is a group of chronic low-grade inflammatory diseases with islet resistance and islet $\beta$-cell dysfunction as the main pathophysiological changes. Insulin resistance and longterm hyperglycaemia have toxic effects on islet $\beta$-cells and gradually decrease islet $\beta$-cell function. ${ }^{12}$ Recently, a study showed that a longer duration of diabetes was associated with a higher risk of insulin resistance. ${ }^{13}$ Studies have found that insulin resistance affects $\mathrm{BC}$, particularly the distribution of fat mass. ${ }^{14}$ Recent studies have shown that impaired glucose control and the course of disease are two major factors affecting $\mathrm{BC}$ in patients with $\mathrm{T} 2 \mathrm{D},{ }^{15,16}$ but studies on whether diabetes duration and glycaemic control impact on these facts have been limited and equivocal with small sample sizes. Considering these results, this study was designed to investigate differences and changing trends among T2D patients in different age groups and the roles of disease duration and glycaemic control in T2D-associated changes in $\mathrm{BC}$ and BMD.

\section{Patients and Methods}

\section{Subjects}

In this cross-sectional study, we screened 7428 individuals between 45 and 85 years of age who met the 1999 World Health Organization (WHO) diagnosis and classification criteria of $\mathrm{T}_{2} \mathrm{D}^{17}$ at the First Affiliated Hospital of Chongqing Medical University from Sep 2013 to
Dec 2018. Of these individuals, 5916 patients were excluded due to unavailable BC and BMD data, and 92 patients were excluded due to meeting exclusion criteria. Exclusion criteria were a history of metabolic bone diseases and conditions affecting nutritional status, such as hyperthyroidism and hypothyroidism, Cushing's syndrome, rheumatoid arthritis, acute inflammatory diseases, autoimmune disease, malignant tumour, chronic liver or renal failure. Eventually, a total of 1474 subjects were enrolled, including 817 males and 657 females (Figure $1)$. None of the subjects had been treated with glucocorticosteroids or anti-obesity drugs within the previous 6 months. All subjects were divided into four groups according to age: the 45-54-years-old group, 55-64-years-old group, 65-74-years-old group, and $\geq 75$-years-old group. Then, the duration of diabetes was divided into three groups according to a statistical method. Informed consent was obtained from all participants. This study was approved by the Ethical Committee of the First Affiliated Hospital of Chongqing Medical University.

\section{Methods}

\section{Anthropometric and Biochemical Measurements}

A variety of anthropometric measurements, including weight and height, were measured while the subjects were in light clothing without shoes. Weight was measured with an electronic scale to the nearest $0.1 \mathrm{~kg}$, and body height was measured with a hypsometer to the nearest $0.1 \mathrm{~cm}$. Body mass index (BMI) was calculated as weight $(\mathrm{kg})$ divided by height $(\mathrm{m})$ squared, and then the BMI classification recommended by the WHO was performed. ${ }^{18}$ Blood pressure was measured by a trained examiner.

Biochemical index measurements, including total cholesterol (TC), triglycerides (TG), low-density lipoprotein cholesterol (LDL-C), and high-density lipoprotein (HDLC), were enzymatically measured by an automatic analyser (Model 7080; Hitachi, Tokyo, Japan). Haemoglobin $A_{1 C}$ $\left(\mathrm{HbA}_{1 \mathrm{c}}\right)$ was measured using a boronate affinity highperformance liquid chromatography (Trinity Biotech, ultra2, Trinity Biotech, Dublin, Ireland).

\section{Measurements of BC and BMD by DEXA}

$\mathrm{BC}$ and $\mathrm{BMD}$ were measured with the same total-body dual-energy X-ray absorptiometry (DEXA) scanner (Hologic Discovery QDR ${ }^{\circledR}$ Series, Bedford, USA). All operations were carried out in accordance with the specifications of the instrument manual. The subject lied in 


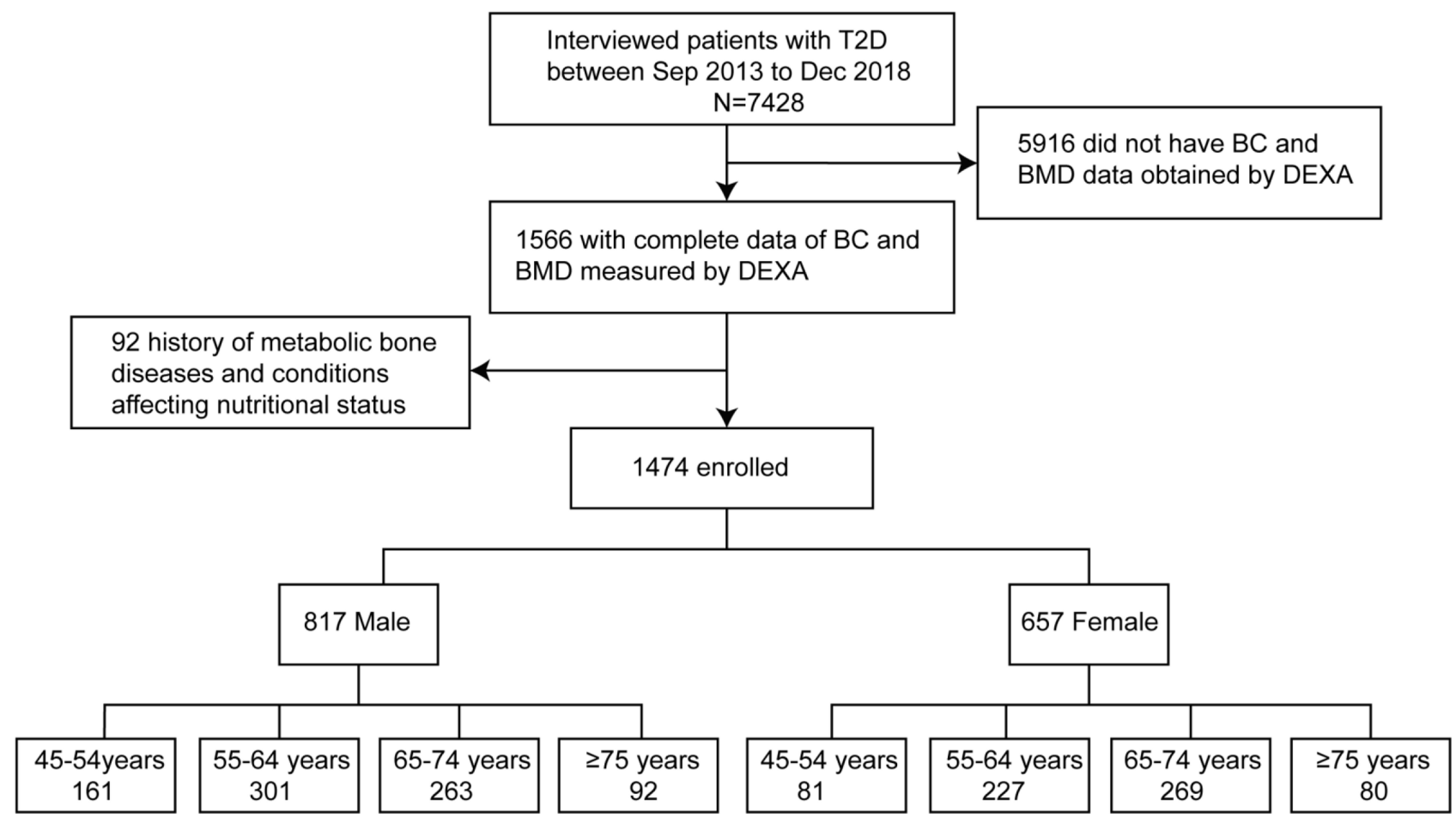

Figure I Flow chart of study population in the study.

Abbreviations: T2D, type 2 diabetes; Sep, September; Dec, December; BC, body composition; BMD, bone mineral density; DEXA, dual-energy X-ray absorptiometry.

a supine position, and was scanned from the head to the feet in the standard mode. The width of the scanning range was fixed at $60 \mathrm{~cm}$, and the scanning time was approximately $20 \mathrm{~min}$. Corresponding data were calculated using the Hologic full-body DEXA reference database. Determination indexes included total fat mass, total lean mass, visceral adipose tissue (VAT), appendage lean mass (ALM), total bone mineral density (TBMD), lumbar spine bone mineral density (LBMD) and femoral neck bone mineral density (FNBMD). The fat mass index (FMI; total fat mass $/$ height $^{2}$ ) and lean mass index (LMI; total lean mass $/$ height $^{2}$ ) were calculated accordingly. In this study, we defined normal density, osteopenia, and osteoporosis as a $\mathrm{T}$-score $\geq-1: 0$, between -1.0 and -2.5 , and $\leq-2.5$, respectively, in accordance with the WHO definitions. $^{19}$

\section{Statistical Analysis}

All data were analysed with SPSS version 22.0 (IBM SPSS, Armonk, New York). The data are presented as the means \pm standard deviations (SDs) for continuous variables and as frequencies (proportions) for categorical variables. Continuous variables were compared using oneway analysis of variance (ANOVA), and categorical variables were compared using the chi-square test Multiple linear regression analysis was performed to evaluate the association between $\mathrm{BC}$ and $\mathrm{BMD}$ and age or duration of diabetes or $\mathrm{HbA}_{1 \mathrm{c}}$. Replicates and data reviews were performed for quality assurance. A two-sided $P$ value of $<0.05$ was considered significant.

\section{Results}

\section{Clinical Characteristics, BC and BMD for Each Sex}

Clinical characteristics of subjects in this study are listed in Table 1. The mean age of enrolled patients was 63.85 \pm 8.71 years, and their mean duration of diabetes was 9.92 \pm 7.05 years. Female patients had a higher mean age and duration of diabetes than males. Male patients had higher heights and weights. The mean BMI of enrolled patients was $24.72 \pm 3.33 \mathrm{~kg} / \mathrm{m}^{2} ; 52.9 \%$ had a normal BMI, $38.5 \%$ were overweight and the remaining $6.0 \%$ were obese. Compared with male patients, female patients had higher systolic blood pressure (SBP) but lower diastolic blood pressure (DBP). The mean $\mathrm{HbA}_{1 \mathrm{c}}$ level was lower in females. There were no differences in TG, TC and LDL$\mathrm{C}$ between sexes, but female patients had higher HDL-C. The proportion of male and female subjects who received 


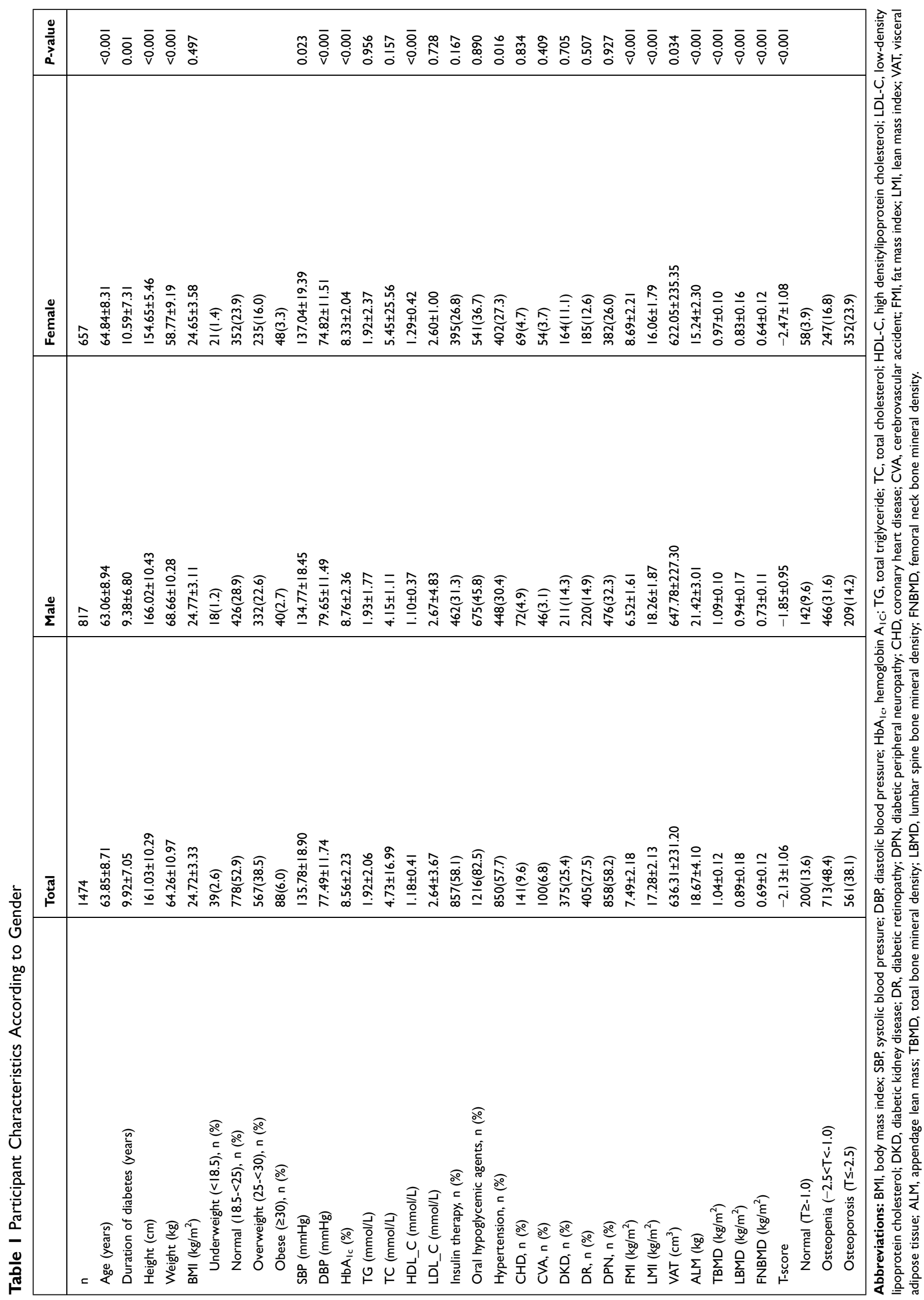


insulin therapy was $31.3 \%$ and $26.8 \%$, respectively; $82.5 \%$ of all subjects took oral hypoglycaemic agents, with no differences between sexes. Furthermore, data regarding the occurrence of diabetes comorbidities and complications are shown in Table 1. Male patients had a higher prevalence of hypertension, but there was no significant difference in the occurrence of other diabetes comorbidities and complications, such as coronary heart disease (CHD) and cerebrovascular accident (CVA), diabetic kidney disease (DKD), diabetic retinopathy (DR) and diabetic peripheral neuropathy (DPN), between patients of either sex.

In terms of $\mathrm{BC}$, compared with female patients, male patients had a lower FMI $(6.52 \pm 1.61$ vs $8.69 \pm 2.21)$ but higher LMI (18.26 \pm 1.87 vs $16.06 \pm 1.79)$, VAT (647.78 \pm 227.30 vs 622.05 \pm 235.35$)$ and ALM $(21.42 \pm 3.01$ vs $15.24 \pm 2.30)$. For BMD, male patients had a higher TBMD ( $1.09 \pm 0.10$ vs $0.97 \pm 0.10)$, LBMD $(0.94 \pm 0.17$ vs $0.83 \pm 0.16)$ and FNBMD $(0.73 \pm 0.11$ vs $0.64 \pm 0.12)$. The proportion of male and female patients who had osteopenia was $31.6 \%$ and $16.8 \%$, respectively, and who had osteoporosis were $14.2 \%$ and $23.9 \%$, respectively.

\section{Differences in BC Parameters Among the Age Groups}

As shown in Table 2, there were sex differences in the changes in FMI and LMI. There was no significant difference in FMI among different age groups of male patients ( $P=0.320)$, and LMI decreased with age $(\mathrm{P}=0.006)$. FMI of females increased with age $(P=0.001)$, and there was no significant difference in LMI among the different age groups ( $P=0.166$ ). In terms of the regional distribution of $\mathrm{BC}$, the variation trend of the different sexes was similar: VAT increased with age $(P=0.002, P<0.001$ for males and females, respectively), and ALM decreased with age (both $P<0.001)$. These trends are shown in Figure 2A-D.

Pearson correlation analysis showed that in male patients, age was negatively associated with LMI ( $r=$ $-0.108, P=0.002$ ), and there was no significant correlation between age and FMI $(r=0.044, P=0.209)$. In female patients, age was positively associated with FMI $(r=0.146$, $P<0.001$ ), but there was no significant correlation between age and LMI ( $r=-0.047, P=0.225)$. In terms of the regional distribution of BC, age was positively associated with VAT ( $r=0.123, r=0.156$, respectively, in males and females, both $P<0.001)$ but negatively associated with ALM $(r=-0.189, r=$
-0.193 , respectively, in males and females, both $P<0.001$ ). These specific data are not shown in the table.

\section{Differences in BMD Among the Age Groups}

As shown in Table 2, there were sex differences in the variation trend of TBMD and BMD in different parts of the body. In male patients, there was no significant difference in TBMD among the age groups $(P=0.325)$, while TBMD decreased with age in female patients $(P<0.001)$. LBMD increased with age in male patients $(P=0.003)$ but decreased with age in female patients $(P<0.001)$. In both male and female patients, FNBMD decreased with age (both $P<0.001$ ). For the change in the T-score, there was no significant difference at each age group in male patients $(P=0.155)$, while the T-score gradually decreased in female patients with age $(P<0.001)$. The prevalence rates of osteopenia and osteoporosis in each age group are shown in Table 2. These trends are shown in Figure 2E-H.

Pearson correlation analysis indicated that age was no significantly correlated with TBMD $(r=-0.038, P=0.278)$ in male patients but was negatively associated with TBMD in females $(r=-0.436, P<0.001)$. Meanwhile, age was positively associated with LBMD $(r=0.133, P<0.001)$ but negatively associated with FNBMD $(r=-0.170, P<0.001)$ in male patients. In female patients, age was negatively associated with LBMD $(r=-0.203, P<0.001)$ and FNBMD $(r=-0.442, P<0.001)$. The T-score was not associated with age in male patients $(r=-0.053, P=0.129)$ but was negatively associated with age in female patients $(r=-0.403$, $P<0.001)$. These specific data are not shown in the table.

\section{Multivariate Analyses}

As shown in Table 3, BC and BMD of different sites were taken as dependent variables and age as an independent variable, respectively, and adjusted for the duration of diabetes, SBP, DBP, $\mathrm{HbA}_{1 \mathrm{c}}$, TG, TC, LDL-C, insulin therapy and hypoglycaemic agents. In terms of $\mathrm{BC}$, age was positively correlated with VAT, negatively correlated with ALM, and was not correlated with FMI and LMI in male patients. Meanwhile, age was positively correlated with FMI and VAT, negatively correlated with ALM, and not correlated with LMI in female patients. In terms of BMD, age was positively correlated with LBMD, negatively correlated with FNBMD and not correlated with 


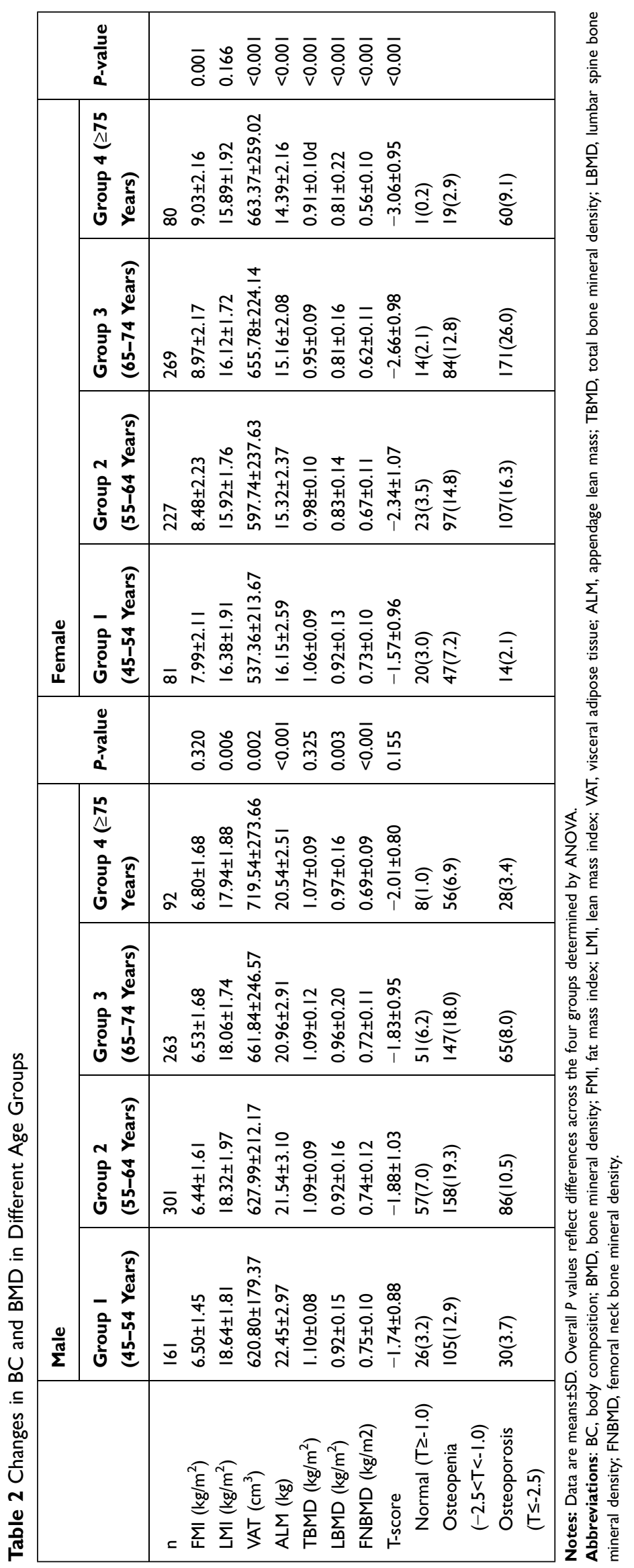


A

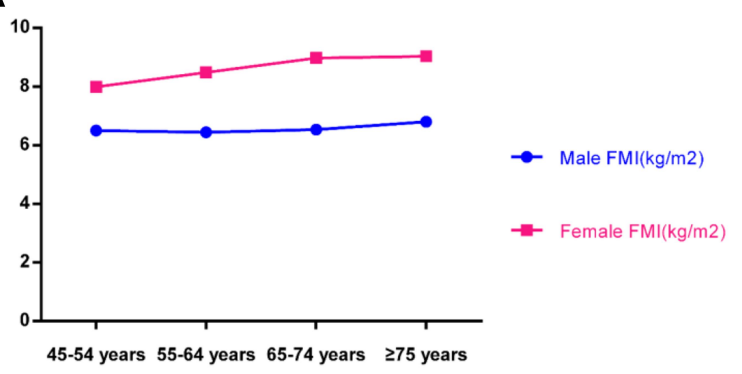

C

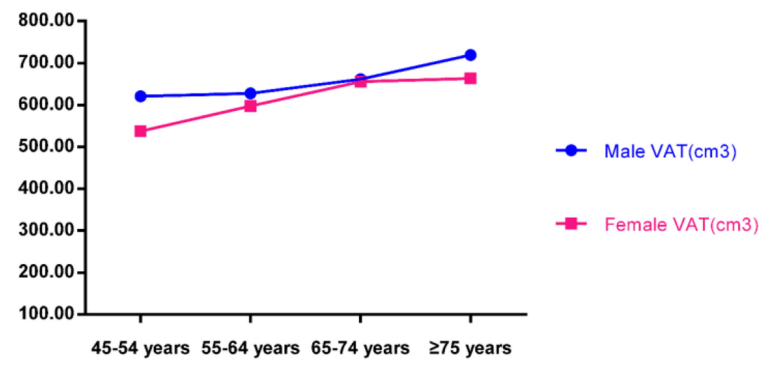

E

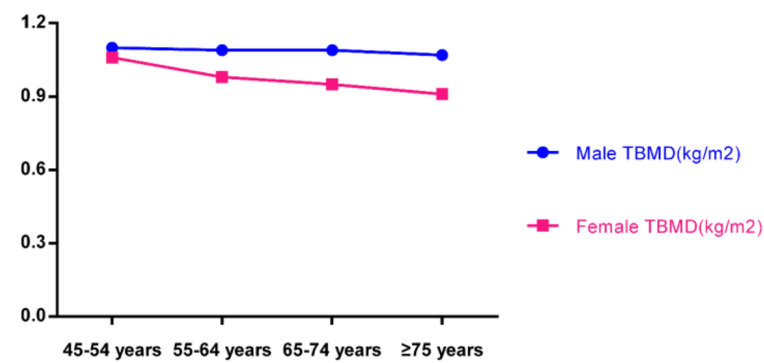

G

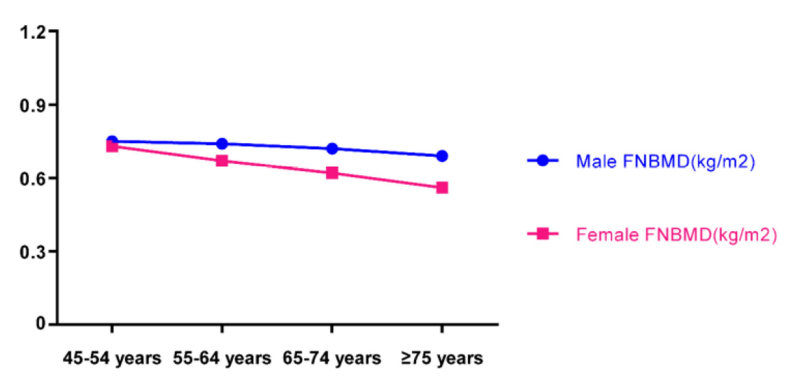

B

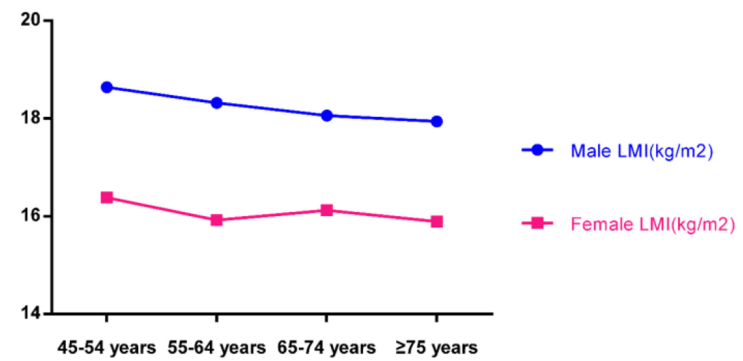

D

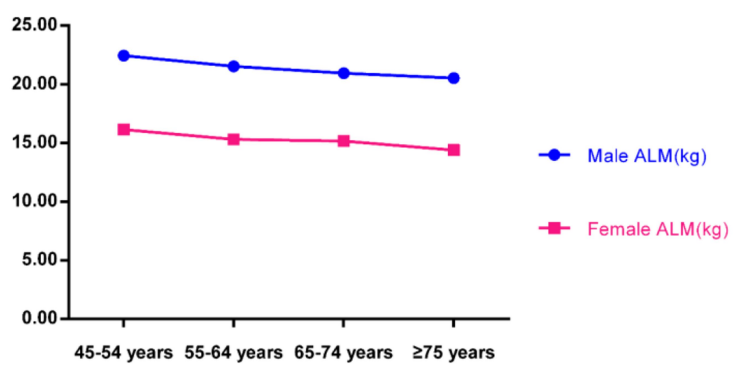

F

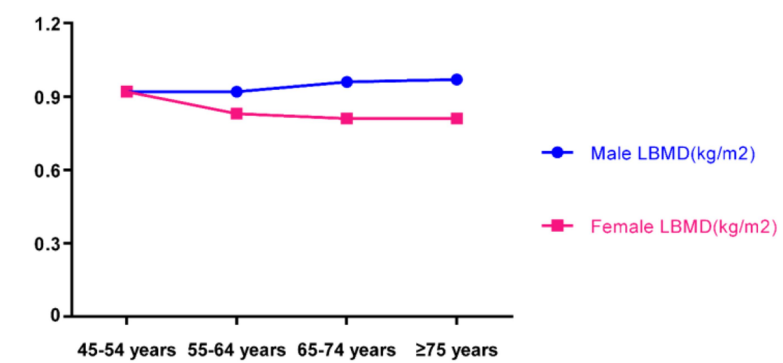

H

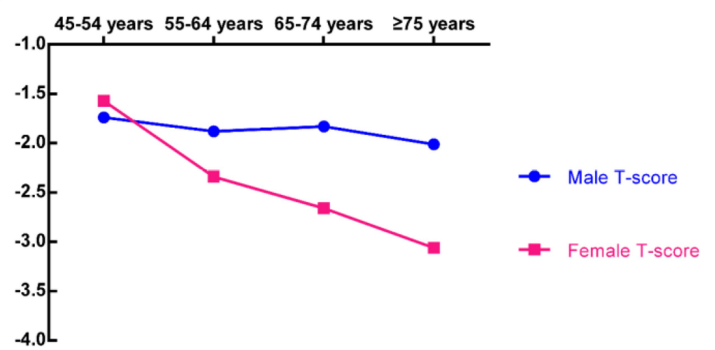

Figure 2 The changes of $B C$ and $B M D$ in different age groups.

Notes: (A) The changes of FMI in different age groups by sex; (B) The changes of LMI in different age groups by sex; (C) The changes of VAT in different age groups by sex;

(D) The changes of ALM in different age groups by sex; (E) The changes of TBMD in different age groups by sex; (F) The changes of LBMD in different age groups by sex; (G) The changes of FNBMD in different age groups by sex; $(\mathbf{H})$ The changes of T-score in different age groups by sex.

Abbreviations: FMI, fat mass index; LMI, lean mass index; VAT, visceral adipose tissue; ALM, appendage lean mass; TBMD, total bone mineral density; LBMD, lumbar spine bone mineral density; FNBMD, femoral neck bone mineral density. 
Table 3 Multiple Linear Regressions with BC and BMD at Different Site as Dependent Variables and Age as Independent Variables

\begin{tabular}{|l|l|l|l|l|l|l|l|l|}
\hline & \multicolumn{7}{l}{ Male } & \multicolumn{2}{l|}{ Female } \\
\hline & $\boldsymbol{\beta}$ & $\mathbf{t}$ & $\boldsymbol{P}$-value & $\mathbf{9 5 \%} \mathbf{C l}$ & $\boldsymbol{\beta}$ & $\mathbf{t}$ & $\boldsymbol{P}$-value & $\mathbf{9 5 \%} \mathbf{C l}$ \\
\hline FMI & 0.068 & 1.588 & 0.113 & $-0.003,0.027$ & 0.155 & 3.435 & 0.001 & $0.018,0.065$ \\
\hline LMI & -0.079 & -1.861 & 0.063 & $-0.034,0.001$ & -0.055 & -1.205 & 0.229 & $-0.031,0.007$ \\
\hline VAT & 0.187 & 4.467 & $<0.001$ & $2.671,6.861$ & 0.172 & 3.847 & $<0.001$ & $2.347,7.242$ \\
\hline ALM & -0.133 & -3.196 & 0.001 & $-0.073,-0.017$ & -0.163 & -3.645 & $<0.001$ & $-0.068,-0.020$ \\
\hline TBMD & -0.021 & -0.484 & 0.629 & $-0.001,0.001$ & -0.476 & -11.552 & $<0.001$ & $-0.007,-0.005$ \\
\hline LBMD & 0.158 & 3.665 & $<0.001$ & $0.001,0.005$ & -0.242 & -5.349 & $<0.001$ & $-0.007,-0.003$ \\
\hline FNBMD & -0.14 & -3.241 & 0.001 & $-0.003,-0.001$ & -0.454 & -10.991 & $<0.001$ & $-0.007,-0.005$ \\
\hline
\end{tabular}

Notes: $\beta$ value means regression coefficient, adjusted the confounders such as duration of diabetes, SBP, DBP, HbA $\mathrm{Ic}_{\mathrm{c}}, \mathrm{TG}, \mathrm{TC}, \mathrm{LDL}-\mathrm{C}$, insulin therapy and hypoglycemic agents.

Abbreviations: FMI, fat mass index; LMI, lean mass index; VAT, visceral adipose tissue; ALM, appendage lean mass; TBMD, total bone mineral density; LBMD, lumbar spine bone mineral density; FNBMD, femoral neck bone mineral density.

TBMD in males. However, in female patients, age was negatively correlated with TBMD, LBMD and FNBMD.

\section{The Role of the Duration of Diabetes and Glycaemic Control}

As shown in Table 4, the duration of diabetes was divided into three groups according to a statistical method. FMI, ALM, TBMD, LBMD and FNBMD showed differences among each group, while LMI, VAT and T-score showed no significant differences. Pearson correlation analysis showed that diabetes duration was positively associated with FMI $(r=0.099$, $P<0.001)$ but negatively associated with ALM $(r=-0.095$, $P<0.001)$, TBMD $(r=-0.075, P=0.004)$, FNBMD $(r=-0.105$,
$P<0.001)$ and the T-score $(r=-0.052, P=0.046)$. These specific data are not shown in the table. Multiple linear regressions with $\mathrm{BC}$ and $\mathrm{BMD}$ at different site as dependent variables and age as independent variables and adjusted for confounding factors are shown in Table 5. Model 1 was adjusted for sex and traditional metabolic and drug factors, including SBP, DBP, $\mathrm{HbA}_{1 \mathrm{c}}$, TG, TC, LDL-C, insulin therapy and hypoglycaemic agents. The duration of diabetes was positively associated with only FMI. However, after further adjusting for age in model 2, the relationship between the duration of diabetes and $\mathrm{BC}$ and $\mathrm{BMD}$ disappeared.

With $\mathrm{HbA}_{1 \mathrm{c}}$ as the independent variable, multiple linear regression analysis was conducted to adjust for confounding

Table 4 Changes in BC and BMD in Different Duration of Diabetes

\begin{tabular}{|c|c|c|c|c|}
\hline Duration of Diabetes (Years) & Low Rank (2.64 \pm 2.08$)$ & Middle Rank (9.37 \pm 1.55$)$ & High Rank ( $18.22 \pm 4.68)$ & $P$-value \\
\hline $\mathrm{n}$ & 503 & 497 & 474 & \\
\hline $\mathrm{FMI}\left(\mathrm{kg} / \mathrm{m}^{2}\right)$ & $7.29 \pm 2.20$ & $7.47 \pm 2.14$ & $7.72 \pm 2.19$ & 0.009 \\
\hline LMI $\left(\mathrm{kg} / \mathrm{m}^{2}\right)$ & $17.33 \pm 2.20$ & $17.22 \pm 2.19$ & $17.26 \pm 1.98$ & 0.787 \\
\hline VAT $\left(\mathrm{cm}^{3}\right)$ & $626.62 \pm 236.61$ & $630.7 I \pm 229.20$ & $652.43 \pm 227.46$ & 0.176 \\
\hline $\mathrm{ALM}(\mathrm{kg})$ & $19.16 \pm 4.10$ & $|8.5| \pm 4.20$ & $|8.26 \pm 3.9|$ & 0.002 \\
\hline TBMD $\left(\mathrm{kg} / \mathrm{m}^{2}\right)$ & $1.05 \pm 0.11$ & $1.03 \pm 0.13$ & $1.03 \pm 0.11$ & 0.044 \\
\hline LBMD $\left(\mathrm{kg} / \mathrm{m}^{2}\right)$ & $0.89 \pm 0.18$ & $0.87 \pm 0.17$ & $0.90 \pm 0.19$ & 0.043 \\
\hline FNBMD $\left(\mathrm{kg} / \mathrm{m}^{2}\right)$ & $0.7 I \pm 0.12$ & $0.69 \pm 0.13$ & $0.68 \pm 0.12$ & 0.001 \\
\hline T-score & $-2.04 \pm 1.02$ & $-2.20 \pm 1.12$ & $-2.13 \pm 1.03$ & 0.052 \\
\hline Normal $(T \geq-I .0)$ & $68(4.6)$ & $68(4.6)$ & $64(4.3)$ & \\
\hline Osteopenia $(-2.5<T<-1.0)$ & $262(17.8)$ & $227(15.4)$ & $224(15.2)$ & \\
\hline Osteoporosis $(\mathrm{T} \leq-2.5)$ & $173(1 \mid .7)$ & $202(13.7)$ & $186(12.6)$ & \\
\hline
\end{tabular}

Notes: Data are means \pm SD. Overall $P$ values reflect differences across the three groups determined by ANOVA.

Abbreviations: FMI, fat mass index; LMI, lean mass index; VAT, visceral adipose tissue; ALM, appendage lean mass; TBMD, total bone mineral density; LBMD, lumbar spine bone mineral density; FNBMD, femoral neck bone mineral density. 
Table 5 Multiple Regression Analysis of the Data on BC and BMD with the Duration of Diabetes as the Independent Variables

\begin{tabular}{|c|c|c|c|c|c|c|}
\hline & $\beta^{\prime}$ & SE & $\beta$ & $\mathbf{t}$ & $P$-value & $95 \% \mathrm{Cl}$ \\
\hline \multicolumn{7}{|l|}{ Modell } \\
\hline FMI & 0.019 & 0.008 & 0.059 & 2.237 & 0.025 & $0.002,0.035$ \\
\hline LMI & 0.007 & 0.008 & 0.023 & 0.904 & 0.366 & $-0.008,0.023$ \\
\hline VAT & 1.575 & 0.998 & 0.048 & 1.578 & 0.115 & $-0.383,3.532$ \\
\hline ALM & -0.022 & 0.012 & -0.038 & -1.929 & 0.054 & $-0.045,0.004$ \\
\hline TBMD & 0.000 & 0.000 & -0.011 & -0.405 & 0.686 & $-0.00 \mathrm{I}, 0.00 \mathrm{I}$ \\
\hline LBMD & 0.001 & 0.001 & 0.042 & 1.412 & 0.158 & $0.000,0.003$ \\
\hline FNBMD & -0.001 & 0.001 & -0.042 & -1.507 & 0.132 & $-0.002,0.000$ \\
\hline \multicolumn{7}{|l|}{ Model2 } \\
\hline FMI & 0.013 & 0.008 & 0.040 & 1.507 & 0.132 & $-0.004,0.029$ \\
\hline LMI & 0.011 & 0.008 & 0.036 & 1.357 & 0.175 & $-0.005,0.027$ \\
\hline VAT & 0.394 & 1.002 & 0.012 & 0.394 & 0.694 & $-1.571,2.359$ \\
\hline ALM & -0.010 & 0.012 & -0.017 & -0.877 & 0.380 & $-0.033,0.013$ \\
\hline TBMD & 0.000 & 0.000 & 0.027 & 1.012 & 0.312 & $0.000,0.001$ \\
\hline LBMD & 0.001 & 0.001 & 0.045 & 1.513 & 0.131 & $0.000,0.003$ \\
\hline FNBMD & 0.000 & 0.000 & 0.007 & 0.271 & 0.787 & $-0.001,0.001$ \\
\hline
\end{tabular}

Notes: Modell was adjusted for gender, traditional metabolic and drug factors, including SBP, DBP, HbAlc, TG, TC, LDL-C, insulin therapy and hypoglycemic agents. Model 2 was further adjusted for age. $\beta$ ' stands for nonstandardized coefficient, $\beta$ stands for standardized coefficient.

Abbreviations: FMI, fat mass index; LMI, lean mass index; VAT, visceral adipose tissue; ALM, appendage lean mass; TBMD, total bone mineral density; LBMD, lumbar spine bone mineral density; FNBMD, femoral neck bone mineral density.

factors (Table 6). Model 1 was adjusted for sex and traditional metabolic and drug factors, including duration of diabetes, SBP, DBP, TG, TC, LDL-C, insulin therapy and hypoglycaemic agents. $\mathrm{HbA}_{1 \mathrm{c}}$ was negatively associated with LMI, ALM and FNBMD. After further adjusting for age in model 2, these relationships still existed.

Table 6 Multiple Regression Analysis of the Data on BC and BMD with $\mathrm{HbA}_{\mathrm{Ic}}$ as the Independent Variables

\begin{tabular}{|c|c|c|c|c|c|c|}
\hline & $\beta$, & SE & $\boldsymbol{\beta}$ & $\mathbf{t}$ & $P$-value & $95 \% \mathrm{Cl}$ \\
\hline \multicolumn{7}{|l|}{ Modell } \\
\hline FMI & -0.018 & 0.025 & -0.018 & -0.731 & 0.465 & $-0.066,0.030$ \\
\hline LMI & -0.087 & 0.023 & -0.090 & -3.694 & $<0.001$ & $-0.133,-0.041$ \\
\hline VAT & -1.838 & 2.956 & -0.018 & -0.622 & 0.534 & $-7.637,3.960$ \\
\hline ALM & -0.227 & 0.034 & -0.123 & -6.574 & $<0.001$ & $-0.294,-0.159$ \\
\hline TBMD & -0.002 & 0.001 & -0.043 & -1.737 & 0.083 & $-0.005,0.000$ \\
\hline LBMD & -0.003 & 0.002 & -0.038 & -1.348 & 0.178 & $-0.007,0.001$ \\
\hline FNBMD & -0.003 & 0.001 & -0.055 & -2.084 & 0.037 & $-0.006,0.000$ \\
\hline \multicolumn{7}{|l|}{ Model2 } \\
\hline FMI & -0.016 & 0.024 & -0.017 & -0.671 & 0.502 & $-0.064,0.032$ \\
\hline LMI & -0.088 & 0.023 & -0.091 & -3.743 & $<0.001$ & $-0.134,-0.042$ \\
\hline VAT & -1.528 & 2.915 & -0.015 & -0.524 & 0.600 & $-7.245,4.190$ \\
\hline ALM & -0.230 & 0.034 & -0.125 & -6.740 & $<0.001$ & $-0.297,-0.163$ \\
\hline TBMD & -0.002 & 0.001 & -0.047 & -1.901 & 0.057 & $-0.005,0.000$ \\
\hline LBMD & -0.003 & 0.002 & -0.038 & -1.359 & 0.175 & $-0.007,0.001$ \\
\hline FNBMD & -0.003 & 0.001 & -0.060 & -2.330 & 0.020 & $-0.006,-0.001$ \\
\hline
\end{tabular}

Notes: Modell was adjusted for gender, traditional metabolic and drug factors, including duration of diabetes, SBP, DBP, TG, TC, LDL-C, insulin therapy and hypoglycemic agents. Model 2 was further adjusted for age. $\beta$ ' stands for nonstandardized coefficient, $\beta$ stands for standardized coefficient.

Abbreviations: FMI, fat mass index; LMI, lean mass index; VAT, visceral adipose tissue; ALM, appendage lean mass; TBMD, total bone mineral density; LBMD, lumbar spine bone mineral density; FNBMD, femoral neck bone mineral density. 


\section{Discussion}

DEXA is a gold standard index for the determination and evaluation of $\mathrm{BC}$. It can not only quantitatively analyse BC (fat and lean mass) and BMD but also evaluate the regional distribution of $\mathrm{BC}$ and $\mathrm{BMD}$. Meanwhile, it has good repeatability and accuracy. In this study, DEXA was used to determine $\mathrm{BC}$ and BMD in T2D patients in Southwest China with a large sample size, and the trend with ageing was discussed. Meanwhile, the roles of disease duration and glycaemic control in T2D-associated changes in $\mathrm{BC}$ and $\mathrm{BMD}$ were assessed. Our results suggested that age, duration of diabetes, and glycaemic control have different effects on $\mathrm{BC}$ and $\mathrm{BMD}$ in T2D.

\section{Effects of Age on BC and BMD in T2D}

$\mathrm{BC}$ and $\mathrm{BMD}$ were influenced by many factors, among which ageing was an important factor. Our findings demonstrated that VAT was positively correlated with age, while ALM was negatively correlated with age in both sexes. These findings are in accordance with previous research demonstrating that during a 6-year follow-up of 2949 Black and white men and women in the Health ABC study, fat mass decreased by $0.066 \mathrm{~kg}$ per year, and ALM decreased by $0.187 \mathrm{~kg}^{3-5}$ Lee et $\mathrm{al}^{20}$ conducted a study of 3153 community residents aged $\geq 65$ years $(7.0 \%$ of males and $6.9 \%$ of females) and found that fat mass, lean mass, and ALM decreased in both sexes after 4 years of followup. Interestingly, we also observed some findings that were not congruent with those of previous studies. The change in FMI in females was positively correlated with age, while the change in LMI was unrelated to age, and the change in FMI or LMI in males was unrelated to age. These findings suggest that age had a relatively small effect on total fat and lean mass and that the change in total fat mass was more pronounced in female patients.

It is generally believed that the effect of ageing on BMD is irreversible. Our data indicated that FNBMD was negatively correlated with age, while there were sex differences in TBMD and LBMD. These parameters decreased in female patients decreased, but changes in TBMD in males were not correlated with age, while LBMD was positively correlated with age. These results are convergent with findings from previous studies. In a study of 358 healthy Chinese men aged 50 to 89 years old, Jiang et $\mathrm{al}^{21}$ found that the BMD of the femoral neck and total hip significantly decreased with increasing age, but there was no significant difference in LBMD. Indeed, other studies have found that LBMD increases with age. In a 10-year follow- up of healthy men, Yoshimura et $\mathrm{al}^{22}$ found that the annual growth rate of LBMD in men over 50 was $0.55 \%$. There was no significant change in LBMD in men over 60, while LBMD decreased by $0.16 \%$ in men over 70 . The increase in LBMD can be explained by age-related bone degeneration, that is, it may be related to the imaging features of lumbar disc degeneration, anterior osteophyte and endplate sclerosis. ${ }^{23}$ As Szulc et $\mathrm{al}^{24}$ found in a cross-sectional study of 934 healthy men between the ages of 19 and 85 that BMD did not significantly increase after 55 years of age (unadjusted). When patients with severe arthritis were excluded, LBMD significantly decreased with age. Hannan et $\mathrm{al}^{25}$ evaluated the BMD of 800 elderly women and men in the Framingham study and found that LBMD decreased with age. There have been relatively few studies on variation trends of BMD with age in T2D patients. According to the variation in BMD in different age groups, targeted and appropriate physical exercise has certain benefits in preventing the occurrence of low bone mass and osteoporosis.

\section{The Effect of the Duration of Diabetes and Glycaemic Control on BC and BMD in $\mathrm{T} 2 \mathrm{D}$}

Interestingly, we found that after adjusting for age, the duration of diabetes was independent of BC and BMD. This suggests that the duration of diabetes did not affect the distribution of $\mathrm{BC}$ or $\mathrm{BMD}$, but the main factor was age. The results are similar to those of a recent study that found the development of metabolic syndrome in patients with T2D might be strongly associated with age rather than glycaemic control or T2D duration. ${ }^{13}$ Another study suggested that there were no significant associations of disease duration and $\mathrm{HbA}_{1 \mathrm{c}}$ with LBMD in patients of both sexes with T2D. ${ }^{26}$ In contrast, Park et $\mathrm{al}^{27}$ evaluated 458 elderly T2D patients aged 70-79 years and 2133 nondiabetic controls and reported that a longer duration of diabetes ( $\geq 6$ years) was associated with a more obvious the decline in muscle function. Another study of a large Chinese cohort with T2D revealed that T2D patients who also fulfilled the criteria for metabolic syndrome tended to have a diabetes duration $\geq 5$ years. ${ }^{21}$ According to a recent report, diabetic duration was an independent risk factor for osteoporosis in postmenopausal women with $\mathrm{T} 2 \mathrm{D} .^{28}$ In general, there have been few reports on the relationship between the course of diabetes and changes in $\mathrm{BC}$ and $\mathrm{BMD}$ to date, and more research is needed to confirm whether diabetes duration has any effect on these parameters. 
Our study also found that a higher $\mathrm{HbA}_{1 \mathrm{c}}$ was associated with a lower LMI and ALM. These results are in accordance with findings from previous research. Park et $\mathrm{al}^{27}$ found that decreased muscle function was associated not only with disease duration but also with higher $\mathrm{HbA}_{1 \mathrm{c}}$ levels $(>8.0 \%)$. Kalyani et $\mathrm{al}^{29}$ screened 5434 nondiabetic participants aged $\geq 50$ years from the National Health and Nutrition Examination Survey (NHANES) and found that higher $\mathrm{HbA}_{1 \mathrm{c}}$ levels in nondiabetic people were also associated with relatively lower lean mass. Additionally, the team of the Baltimore longitudinal study of ageing (2003-2011) that included 984 of the participants aged between 25 and 96 found that after 0-7.5 years of long term follow-up (mean 1.9-2.2 years), higher blood glucose levels indicated a sustained decline in lean mass. ${ }^{30}$ This suggests that hyperglycaemia may contribute to an accelerated decline in lean mass in patients with T2D.

In this study, DEXA was used to analyse the correlation between age and $\mathrm{BC}$ and BMD in a large sample size of T2D patients in Southwest China, providing clinical reference data for changes in $\mathrm{BC}$ and BMD in T2D patients in different age groups. However, the following limitations should be noted. First, subjects in our study were selected from a single centre, and our results may not represent the whole population. Second, this study adopted a cross-sectional design. Therefore, it was difficult to determine a clear cause-andeffect relationship, which needs to be confirmed by more prospective studies with a multicentre population.

\section{Conclusion}

In summary, there are gender differences in the effects of age on $\mathrm{BC}$ and BMD. In addition, changes in $\mathrm{BC}$ and $\mathrm{BMD}$ were not related to the duration of diabetes, and the effect of $\mathrm{HbA}_{1 \mathrm{c}}$ on $\mathrm{BC}$ and $\mathrm{BMD}$ mainly reflected that higher $\mathrm{HbA}_{1 \mathrm{c}}$ was associated with lower lean mass.

\section{Data Sharing Statement}

The datasets used and/or analyzed during the current study are available from the corresponding author upon reasonable request.

\section{Ethics Approval and Informed Consent}

The study protocol was approved by the Ethics Committee of the First Affiliated Hospital of Chongqing Medical University [2018 Scientific Research Ethics (2018-042)]. Written informed consent was obtained from all individual participants included in the study. This study was conducted in accordance with the Declaration of Helsinki. All authors and individual participants provided consent for publication.

\section{Acknowledgments}

We thank the Chongqing medical university for their support towards publishing this article. We also thank the staff and patients of the First Affiliated Hospital of Chongqing Medical University for their contributions during the study. Finally, we thank Xinlu Lv, Li Zheng, Yangmei Zhou, Qinying Zhao and Wenjin Luo for helping with data collection for the study.

\section{Author Contributions}

All authors made substantial contributions to conception and design, acquisition of data, or analysis and interpretation of data; took part in drafting the article or revising it critically for important intellectual content; agreed to submit to the current journal; gave final approval of the version to be published; and agree to be accountable for all aspects of the work. First authors: Ying Tang and Lilin Gong.

\section{Funding}

This work was supported by the National Natural Science Foundation of China (grant number 81800757); the Technological Innovation and Application Development Project of Chongqing (grant number cstc2019iscxmsxmX0207); Chongqing Overseas Students Entrepreneurship and innovation support program (grant number cx2019032); Chongqing Science and Health Joint Medical Research Project (grant number 2018GDRC004); High-end Medical Talent of Middle-aged and Young People in Chongqing (yuweiren (2015)49); International Diabetes Communication and Practice Foundation.

\section{Disclosure}

The authors report no conflicts of interest in this work.

\section{References}

1. Bacchi E, Negri C, Tarperi C, et al. Relationships between cardiorespiratory fitness, metabolic control, and fat distribution in type 2 diabetes subjects. Acta Diabetol. 2014;51(3):369-375. doi:10.1007/ s00592-013-0519-1

2. Gelber RP, Gaziano JM, Orav EJ, Manson JE, Buring JE, Kurth T. Measures of obesity and cardiovascular risk among men and women. J Am Coll Cardiol. 2008;52(8):605-615. doi:10.1016/j.jacc.2008.03.066

3. Koster A, Visser M, Simonsick EM, et al. Association between fitness and changes in body composition and muscle strength. $J$ Am Geriatr Soc. 2010;58(2):219-226. doi:10.1111/j.1532-5415.2009.02681.x 
4. Kyle UG, Genton L, Hans D, Karsegard L, Slosman DO, Pichard C. Age-related differences in fat-free mass, skeletal muscle, body cell mass and fat mass between 18 and 94 years. Eur J Clin Nutr. 2001;55 (8):663-672. doi:10.1038/sj.ejcn.1601198

5. Park SW, Goodpaster BH, Lee JS, et al. Excessive loss of skeletal muscle mass in older adults with type 2 diabetes. Diabetes Care. 2009;32(11):1993-1997. doi:10.2337/dc09-0264

6. Jiang Y, Zhang Y, Jin M, Gu Z, Pei Y, Meng P. Aged-related changes in body composition and association between body composition with bone mass density by body mass index in Chinese han men over 50-year-old. PLoS One. 2015;10(6):e0130400. doi:10.1371/journal. pone. 0130400

7. Goodpaster BH, Krishnaswami S, Resnick H, et al. Association between regional adipose tissue distribution and both type 2 diabetes and impaired glucose tolerance in elderly men and women. Diabetes Care. 2003;26(2):372-379. doi:10.2337/diacare.26.2.372

8. Choi SI, Chung D, Lim JS, et al. Relationship between regional body fat distribution and diabetes mellitus: 2008 to 2010 Korean national health and nutrition examination surveys. Diabetes Metab J. 2017;41 (1):51-59. doi:10.4093/dmj.2017.41.1.51

9. Kim KS, Park KS, Kim MJ, Kim SK, Cho YW, Park SW. Type 2 diabetes is associated with low muscle mass in older adults. Geriatr Gerontol Int. 2014;14(Suppl 1):115-121. doi:10.1111/ggi.12189

10. Kim TN, Park MS, Yang SJ, et al. Prevalence and determinant factors of sarcopenia in patients with type 2 diabetes: the Korean Sarcopenic Obesity Study (KSOS). Diabetes Care. 2010;33(7):1497-1499. doi: $10.2337 / \mathrm{dc} 09-2310$

11. Abdulameer SA, Sulaiman SAS, Hassali MAA, Subramaniam K, Sahib MN. Osteoporosis and type 2 diabetes mellitus: what do we know, and what we can do? Patient Prefer Adherence. 2012;6:435-448. doi:10.2147/PPA.S32745

12. Motahari-Tabari N, Ahmad Shirvani M, Shirzad-e-Ahoodashty M, Yousefi-Abdolmaleki E, Teimourzadeh M. The effect of 8 weeks aerobic exercise on insulin resistance in type 2 diabetes: a randomized clinical trial. Glob J Health Sci. 2014;7(1):115-121. doi:10.5539/gjhs.v7n1p115

13. Abulmeaty MMA, Aljuraiban GS, Alaidarous TA, Alkahtani NM. Body composition and the components of metabolic syndrome in type 2 diabetes: the roles of disease duration and glycemic control. Diabetes Metab Syndr Obes. 2020;13:1051-1059. doi:10.2147/DMSO.S248842

14. Miyazaki Y, Glass L, Triplitt C, Wajcberg E, Mandarino LJ, DeFronzo RA. Abdominal fat distribution and peripheral and hepatic insulin resistance in type 2 diabetes mellitus. Am J Physiol Endocrinol Metab. 2002;283(6):E1135-1143. doi:10.1152/ajpendo.0327.2001

15. Jiang Y, Tan S, Wang Z, Guo Z, Li Q, Wang J. Aerobic exercise training at maximal fat oxidation intensity improves body composition, glycemic control, and physical capacity in older people with type 2 diabetes. J Exerc Sci Fit. 2020;18(1):7-13. doi:10.1016/j.jesf.2019.08.003

16. Ji M, Ren D, Dunbar-Jacob J, Gary-Webb TL, Erlen JA. Correlates of self-management behaviors, glycemic control and metabolic syndrome in patients with type 2 diabetes. Nurs Res. 2020;69(2):E9E17. doi:10.1097/NNR.0000000000000401
17. Poynten AM, Markovic TP, Maclean EL, et al. Fat oxidation, body composition and insulin sensitivity in diabetic and normoglycaemic obese adults 5 years after weight loss. Int J Obes Relat Metab Disord. 2003;27(10):1212-1218. doi:10.1038/sj.ijo.0802393

18. World Health Organisation. Obesity: Preventing and Managing the Global Epidemic. Report of a WHO Consultation (WHO Technical Report Series 894). 2000:1-253

19. Kanis JA, McCloskey EV, Johansson H, Oden A, Melton LJ, Khaltaev N. A reference standard for the description of osteoporosis. Bone. 2008;42 (3):467-475. doi:10.1016/j.bone.2007.11.001

20. Lee JS, Auyeung TW, Leung J, Kwok T, Leung PC, Woo J. The effect of diabetes mellitus on age-associated lean mass loss in 3153 older adults. Diabet Med. 2010;27(12):1366-1371. doi:10.1111/ j.1464-5491.2010.03118.x

21. Jing Y, Hong T, Bi Y, et al. Prevalence, treatment patterns and control rates of metabolic syndrome in a Chinese diabetic population: China cardiometabolic registries 3B study. J Diabetes Investig. 2018;9 (4):789-798. doi:10.1111/jdi.12785

22. Yoshimura N, Kinoshita H, Danjoh S, et al. Bone loss at the lumbar spine and the proximal femur in a rural Japanese community,1990-2000: the Miyama study. Osteoporos Int. 2002;13(10):803-808. doi:10.1007/s001980200111

23. Karabulut O, Tuncer MC, Karabulut Z, Aclkgoz A, Hatipoglu ES, Akkus Z. Relationship between radiographic features and bone mineral density in elderly male. Folia Morphol (Warsz). 2010;69(3):170-176.

24. Szulc P, Marchand F, Duboeuf F, Delmas PD. Cross-sectional assessment of age-related bone loss in men: the MINOS study. Bone. 2000;26(2):123-129. doi:10.1016/s8756-3282(99)00255-0

25. Hannan MT, Felson DT, Dawson-Hughes B, et al. Risk factors for longitudinal bone loss in elderly men and women: the framingham osteoporosis study. J Bone Miner Res. 2010;15(4):710-720. doi:10.1359/jbmr.2000.15.4.710

26. Yao X, Xu X, Jin F, Zhu Z. The correlation of type 2 diabetes status with bone mineral density in middle-aged adults. Diabetes Metab Syndr Obes. 2020;13:3269-3276. doi:10.2147/DMSO.S268592

27. Park SW, Goodpaster BH, Strotmeyer ES, et al. Decreased muscle strength and quality in older adults with type 2 diabetes: the health, aging, and body composition study. Diabetes. 2006;55(6):1813-1818. doi: $10.2337 / \mathrm{db} 05-1183$

28. Zhao X, Yu X, Zhang X. Association between uric acid and bone mineral density in postmenopausal women with type 2 diabetes mellitus in China: a cross-sectional inpatient study. J Diabetes Res. 2020;2020:3982831. doi:10.1155/2020/3982831

29. Kalyani RR, Tra Y, Egan JM, Ferrucci L, Brancati F. Hyperglycemia is associated with relatively lower lean body mass in older adults. $J$ Nutr Health Aging. 2014;18(8):737-743. doi:10.1007/s12603-014-0538-9

30. Kalyani RR, Metter EJ, Egan J, Golden SH, Ferrucci L. Hyperglycemia predicts persistently lower muscle strength with aging. Diabetes Care. 2015;38(1):82-90. doi:10.2337/dc14-1166

\section{Publish your work in this journal}

Diabetes, Metabolic Syndrome and Obesity: Targets and Therapy is an international, peer-reviewed open-access journal committed to the rapid publication of the latest laboratory and clinical findings in the fields of diabetes, metabolic syndrome and obesity research. Original research, review, case reports, hypothesis formation, expert opinion and commentaries are all considered for publication. The manuscript management system is completely online and includes a very quick and fair peer-review system, which is all easy to use. Visit http://www.dovepress.com/testimonials.php to read real quotes from published authors. 